\section{Post impact analysis of damaged variable-stiffness curved composite plates}

\section{B. Arachchige}

\section{H. Ghasemneja}

Hessam.Ghasemnejad@cranfield.ac.uk

Centre for Structures, Assembly and Intelligent Automation, Cranfield University, MK43 0AL, UK

${ }^{*}$ Corresponding author

\title{
Abstract
}

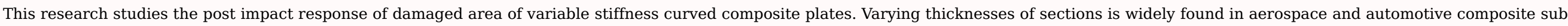

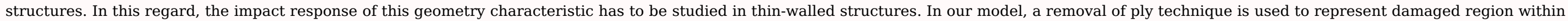

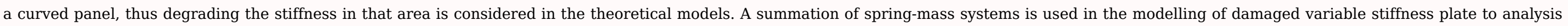

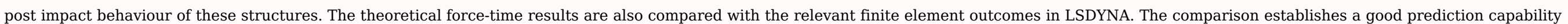
of the proposed model.

Keywords: Polymer-matrix composites (PMCs); Post impact; Variable stiffness; Damage

\section{Nomenclature}

$F(t)$

contact force of undamaged plate

$F(t)_{\text {damaged }}$

contact force of damaged plate

Young's modulus (GPa)

$G_{12}$

Shear modulus (GPa)

Poisson's ratio of plate/impactor

density of plate/impactor

$\mathrm{b}$

width of plate 
$\mathrm{M}_{2}$

mass of impactor

$\mathrm{K}_{1}$

stiffness constant of plate

$\mathrm{K}_{1 \text { (damaged) }}$

damaged stiffness constant of the plate

$\mathrm{K}_{2}$

stiffness constant of striker

$K_{2}^{*}$

effective contact stiffness

$\mathrm{V}$

impact velocity

$t_{m}$

thickness of variable stiffness section

$\mathrm{t}$

time variable

$\omega_{e q}$

equivalent natural frequency of variable stiffness plate

$\omega_{\text {eq(damaged })}$

equivalent natural frequency of variable stiffness plate (damage induced)

\section{Introduction}

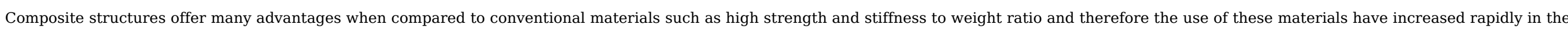

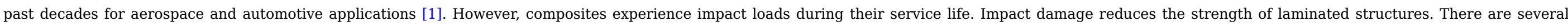

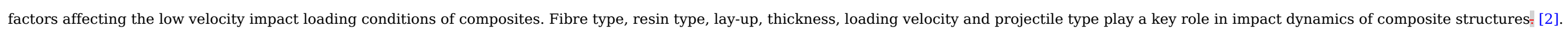

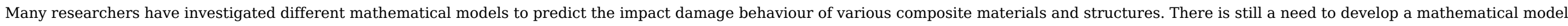

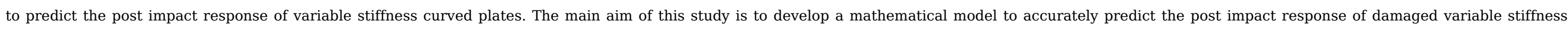
curved composite panels. The most recent models which are used to study the impact behaviour of composite structures are highlighted below.

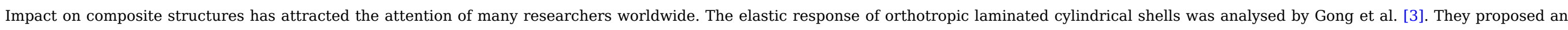




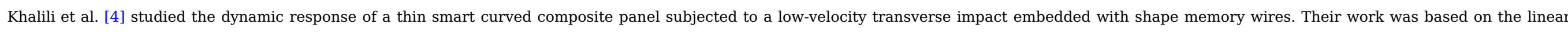

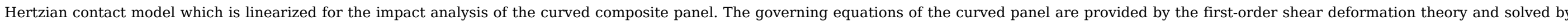

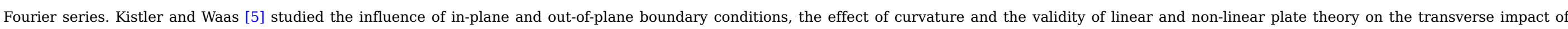

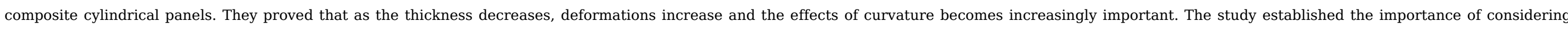

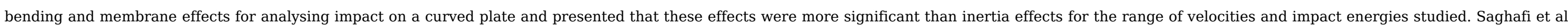

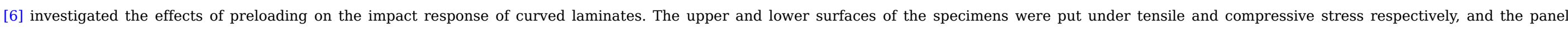

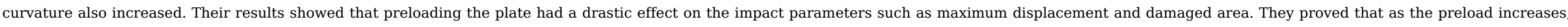

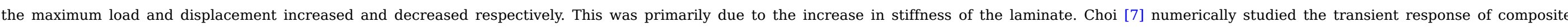

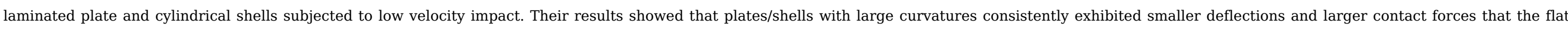

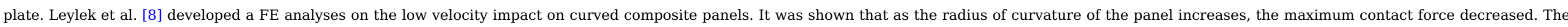

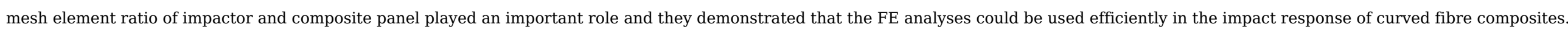

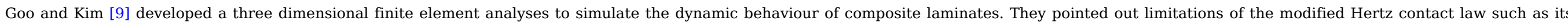

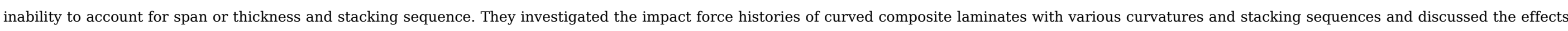
of curvature on the impact behaviour of composite laminates.

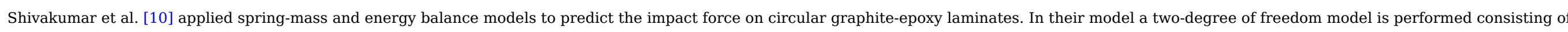

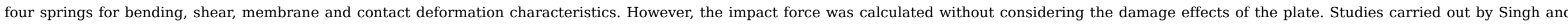

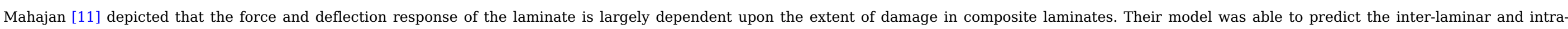

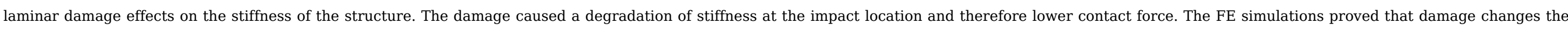

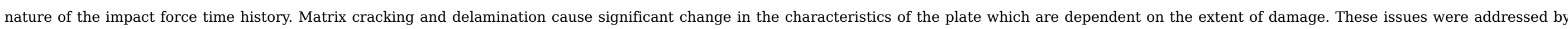

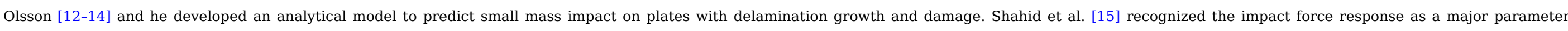

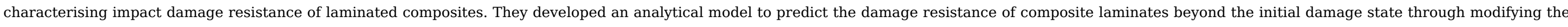
spring constant in Hertzian contact law which is a function of the extent of damage in composites.

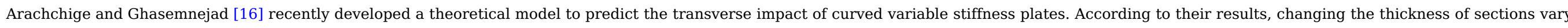

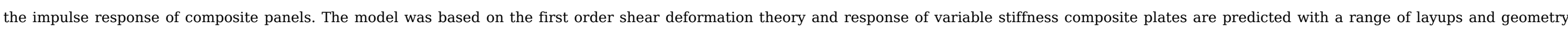
design under low velocity impact loading conditions.

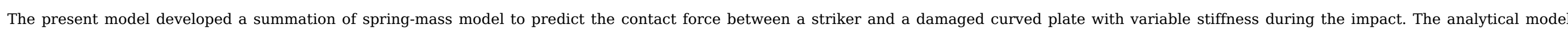

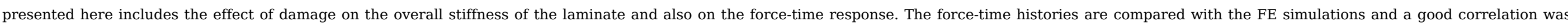
established.

\section{Analytical impact modelling}

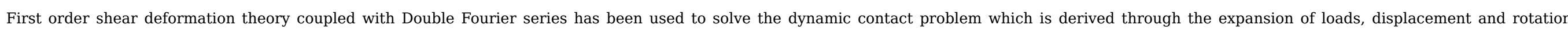

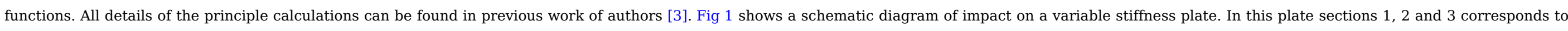
variable stiffness sections with different thicknesses Fig. 2. 
Fig. 1 Schematic diagram of damaged curved composite plate with variable thickness (stiffness).

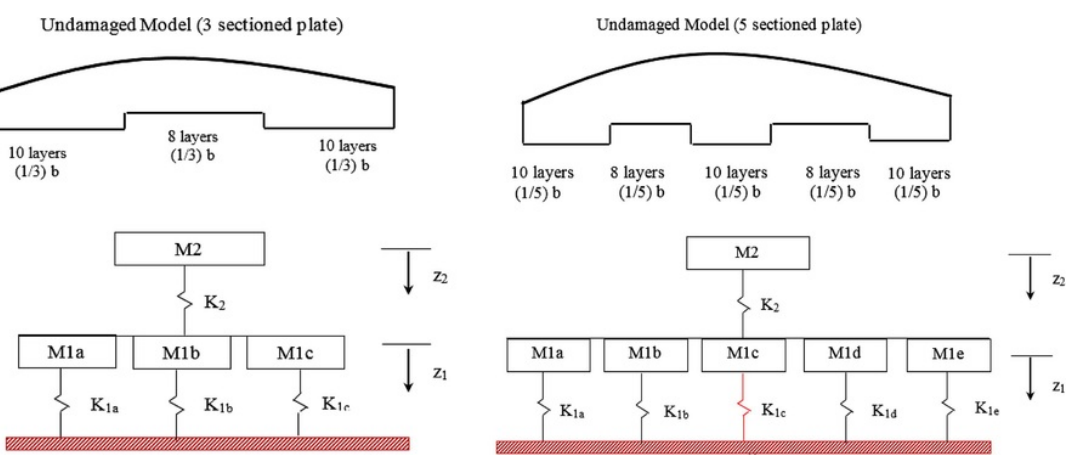

Fig. 2 Mass-Spring diagram of (a) three section plate (b) five section plate.

Love's equations of motion for a curved shell of dimensions a and b, radius $\mathrm{R}$ and thickness $\mathrm{h}$ under external loads [4] are expressed as:

$$
\begin{aligned}
& \frac{\partial N_{x}}{\partial x}+\frac{1}{R} \frac{\partial N_{x \theta}}{\partial \theta}+q_{x}(x, \theta, t)=\rho h \ddot{u} \\
& \frac{\partial N_{x \theta}}{\partial x}+\frac{1}{R} \frac{\partial N_{\theta}}{\partial \theta}+\frac{Q_{\theta}}{R}+q_{\theta}(x, \theta, t)=\rho h \ddot{v} \\
& \frac{\partial Q_{x}}{\partial x}+\frac{1}{R} \frac{\partial Q_{\theta}}{\partial \theta}-\frac{N_{\theta}}{R}+q_{n}(x, \theta, t)=\rho h \ddot{w} \\
& \frac{\partial M_{x}}{\partial x}+\frac{1}{R} \frac{\partial M_{x \theta}}{\partial \theta}-Q_{x}=\frac{\rho h^{3}}{12} \ddot{\beta}_{x} \\
& \frac{\partial M_{x \theta}}{\partial x}+\frac{1}{R} \frac{\partial M_{\theta}}{\partial \theta}-Q_{\theta}=\frac{\rho h^{3}}{12} \ddot{\beta}_{\theta}
\end{aligned}
$$

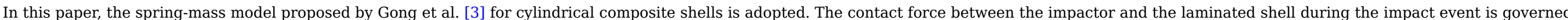
through the Hertzian theory which is defined by,

$F_{c}(t)=K_{2} \partial^{p}$

The force function derived by Gong et al. [3] is defined by;

$F_{c}^{*}(t)=K_{2}^{*}\left[A_{1}\left(C_{1}-1\right) \sin \omega_{1} t+A_{2}\left(C_{2}-1\right) \sin \omega_{2} t\right]$

This approach uses an effective contact stiffness, $K_{2}^{*}$ which results in obtaining an analytical solution for the impact force since Eq. (6) possesses a risk due to its high nonlinearity $K_{2}^{*}=\sqrt{\pi} \Gamma\left(\frac{p+1}{2}\right) \frac{2 \Gamma\left(\frac{p}{2}+1\right)+\sqrt{\pi} \Gamma\left(\frac{p+1}{2}\right)}{4 \Gamma^{2}\left(\frac{p}{2}+1\right)+\pi \Gamma^{2}\left(\frac{p+1}{2}\right)} \delta_{m}^{p-1} K_{2}$ 


$$
\begin{aligned}
& \omega_{1,2}^{2}=\frac{1}{2}\left(\frac{k_{1}+k_{2}}{M_{1}}+\frac{k_{2}}{M_{2}}\right) \mp \sqrt{\frac{1}{4}\left(\frac{k_{1}+k_{2}}{M_{1}}+\frac{k_{2}}{M_{2}}\right)^{2}-\frac{k_{1} k_{2}}{M_{1} M_{2}}} \\
& C_{1}=\frac{k_{2}}{k_{2}-\omega_{1}^{2} M_{2}} \\
& C_{2}=\frac{k_{2}}{k_{2}-\omega_{2}^{2} M_{2}} \\
& A_{1}=\frac{V}{\omega_{1}\left(C_{1}-C_{2}\right)} \\
& A_{1}=\frac{V}{\omega_{2}\left(C_{2}-C_{1}\right)}
\end{aligned}
$$

\section{Analytical modelling of a variable stiffness curved plate (undamaged plate)}

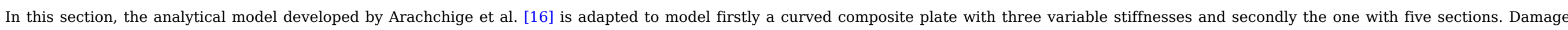
models were also developed for these two models. The material properties of the plate and the impactor are given in Tables 1 and 2.

Table 1 Properties of steel impactor [3].

\begin{tabular}{|l|l|l|}
\hline Young's Modulus & $207 \mathrm{GPa}$ \\
\hline Poisson's ratio & 0.29 \\
\hline Density & $7900 \mathrm{~kg} / \mathrm{m}^{3}$ \\
\hline Diameter & $20 \mathrm{~mm}$ \\
\hline Mass of the striker & $3 \mathrm{~kg}$ \\
\hline Velocity of striker & $6 \mathrm{~m} / \mathrm{s}$ \\
\hline
\end{tabular}

Table 2 Properties of CFRP composites [3].

\begin{tabular}{|l|l|}
\hline $\mathrm{E}_{1}$ & $141 \mathrm{GPa}$ \\
\hline $\mathrm{E}_{2}=\mathrm{E}_{3}$ & $9.7 \mathrm{GPa}$ \\
\hline $\mathrm{G}_{12}=\mathrm{G}_{13}$ & $5.5 \mathrm{GPa}$ \\
\hline $\mathrm{G}_{23}$ & $3.7 \mathrm{GPa}$ \\
\hline $\mathrm{v}_{12}=\mathrm{v}_{13}=\mathrm{v}_{23}$ & 0.29 \\
\hline Density & $1530 \mathrm{~kg} / \mathrm{m}$ \\
\hline
\end{tabular}

The equations for the natural frequency, $\omega_{e q}$ derived in $[4,16]$ to incorporate variable stiffness sections and derived as:

$\omega_{e q}=\int_{0}^{b} \sqrt{\sum_{n=1}^{m} \frac{-\left(C_{13} K_{U}+C_{23} K_{V}+C_{33}+C_{34} K_{X}+C_{35} K_{Y}\right)_{m}}{\rho t_{m}}}$ 


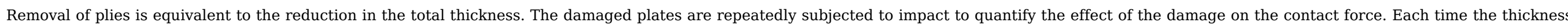

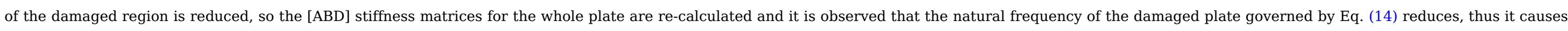
degradation of stiffness.

\section{Analytical modelling of a variable stiffness curved plate (damaged plate)}

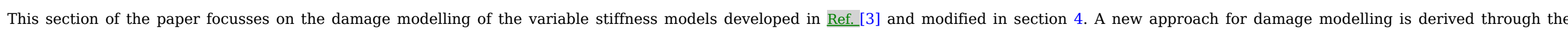

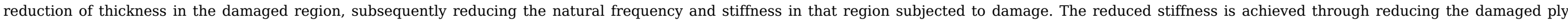

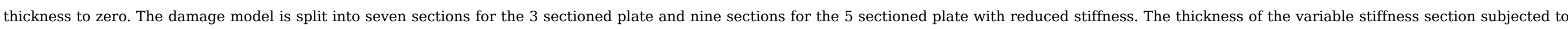

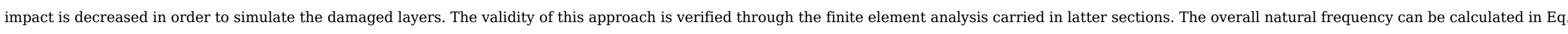

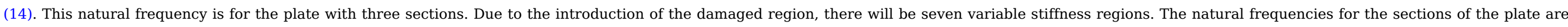

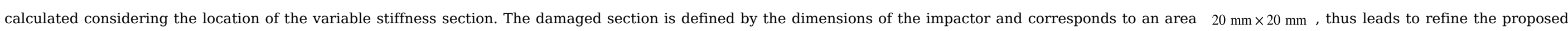
model. This damage model assumes that the damage caused by the spherical impactor which is equivalent to a square region of $20 \mathrm{~mm} \times 20 \mathrm{~mm}$ (see Fig. 3 ).
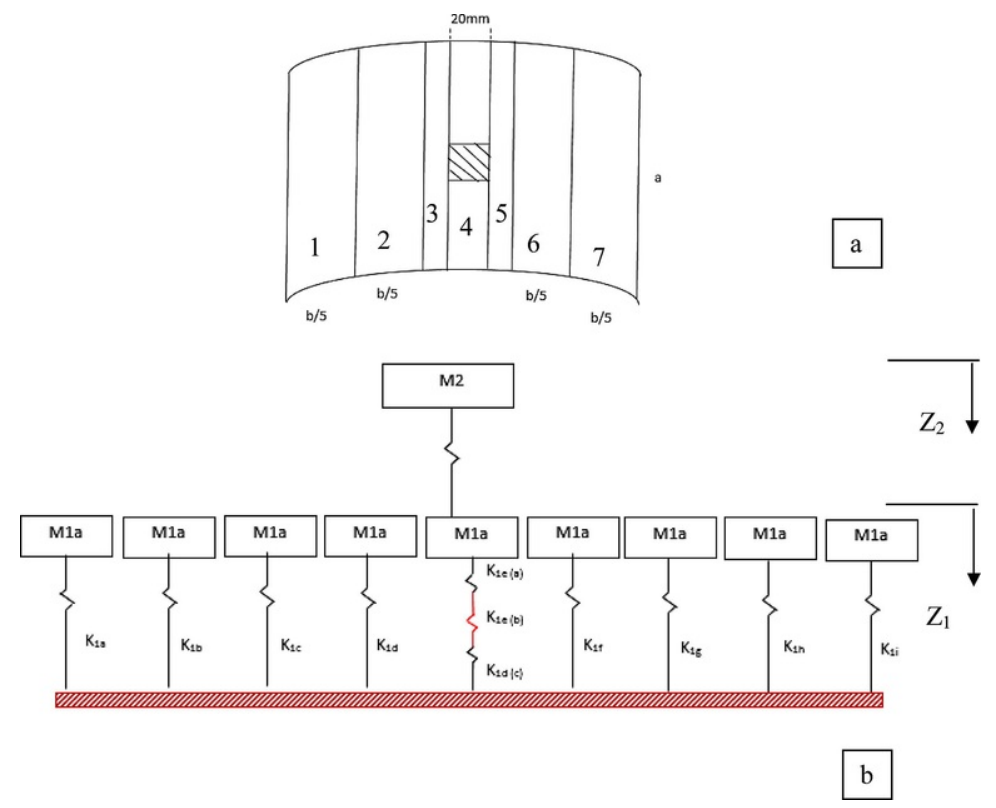

Fig. 3 Two-degrees-freedom spring mass model of damaged 5 sectioned variable thickness plate.

The equivalent natural frequency of Section $4 \omega_{e q(4)}$ is defined as:

$\omega_{e q(4)}=\frac{1}{\omega_{e q(4 a)}}+\frac{1}{\omega_{e q(4 b)}}+\frac{1}{\omega_{e q(4 c)}}$

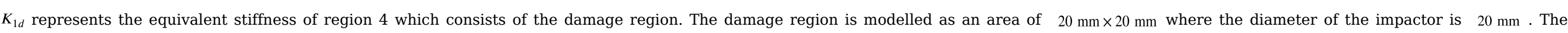
expression of the equivalent stiffness of region 4 is expressed as:

$K_{1 d}=\frac{1}{K_{1 d} a}+\frac{1}{K_{1 d} b}+\frac{1}{K_{1 d} c}$ 
In this model the stiffness constants $K_{1 d} a$ and $K_{1 d} c$ represents stiffnesses of undamaged regions in Section 4 . Therefore, in this model the damage region is refined.

\section{Results and discussions}

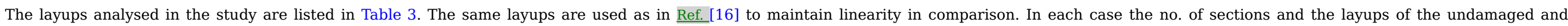

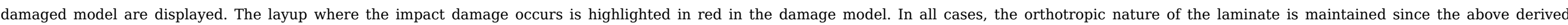

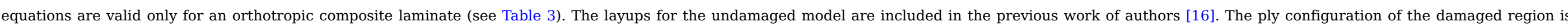
highlighted.

Table 3 Various layups for the variable curved composite panels [3]

\begin{tabular}{|c|c|c|c|}
\hline Layup & No. of sections (Undamaged Model) & No. of Sections (Damaged Model) & Layup (Damaged Model) \\
\hline 1 & 3 & 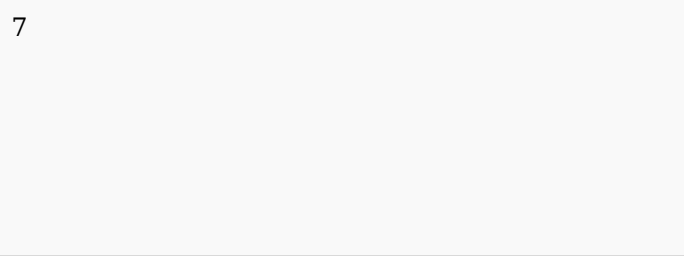 & 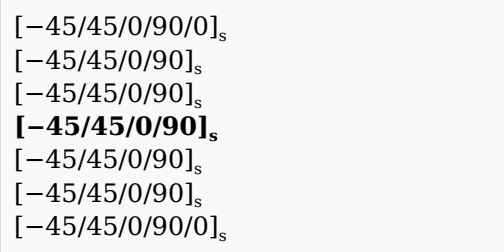 \\
\hline 2 & 3 & 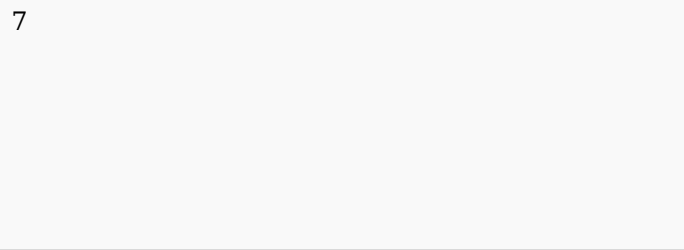 & $\begin{array}{l}{[0 / 90 / 0 / 45 /-45]_{\mathrm{s}}} \\
{[0 / 90 /-45 / 45]_{\mathrm{s}}} \\
{[0 / 90 /-45 /-45]_{\mathrm{s}}} \\
{[\mathbf{0 0 / 9 0 / - 4 5 / - 4 5}]_{\mathrm{s}}} \\
{[0 / 90 /-45 /-45]_{\mathrm{s}}} \\
{[0 / 90 /-45 / 45]_{\mathrm{s}}} \\
{[0 / 90 / 0 / 45 /-45]_{\mathrm{s}}}\end{array}$ \\
\hline 3 & 3 & 7 & $\begin{array}{l}{[0 / 90 / 0 / 90 / 0]_{\mathrm{s}}} \\
{[0 / 90 / 0 / 90]_{\mathrm{s}}} \\
{[0 / 90 / 0 / 90]_{\mathrm{s}}} \\
\mathbf{[ 0 / 9 0 / 0 / 9 0 ] _ { s }} \\
{[0 / 90 / 0 / 90]_{\mathrm{s}}} \\
{[0 / 90 / 0 / 90]_{\mathrm{s}}} \\
{[0 / 90 / 0 / 90 / 0]_{\mathrm{s}}}\end{array}$ \\
\hline 4 & 5 & 9 & $\begin{array}{l}{[-45 / 45 / 0 / 90 / 0]_{\mathrm{s}}} \\
{[-45 / 45 / 0 / 90]_{\mathrm{s}}} \\
{[-45 / 45 / 0 / 90 / 0]_{\mathrm{s}}} \\
{[-45 / 45 / 0 / 90 / 0]_{\mathrm{s}}} \\
{\left[\mathbf{- 4 5 / 4 5 / 0 / 9 0 / 0 ] _ { \mathrm { s } }}\right.} \\
{[-45 / 45 / 0 / 90 / 0]_{\mathrm{s}}} \\
{[-45 / 45 / 0 / 90 / 0]_{\mathrm{s}}} \\
{[-45 / 45 / 0 / 90]_{\mathrm{s}}} \\
{[-45 / 45 / 0 / 90 / 0]_{\mathrm{s}}}\end{array}$ \\
\hline 5 & 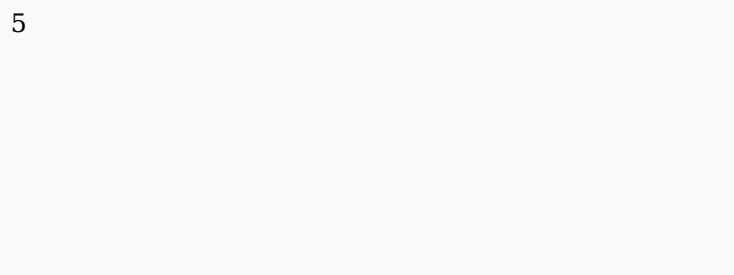 & 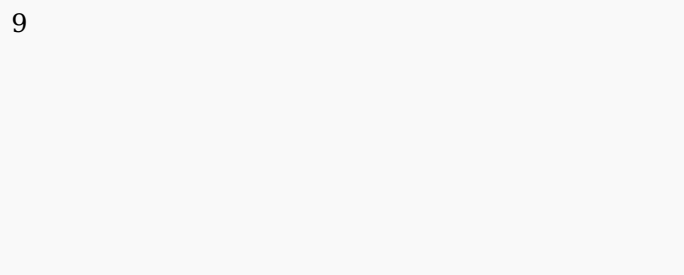 & $\begin{array}{l}{[0 / 90 / 0 / 45 / 45]_{\mathrm{s}}} \\
{[0 / 90 /-45 / 45]_{\mathrm{s}}} \\
{[0 / 90 / 0 / 45 /-45]_{\mathrm{s}}} \\
{[0 / 90 / 0 / 45 /-45]_{\mathrm{s}}} \\
\mathbf{[ 0 / 9 0 / 0 / 4 5 / - 4 5}]_{\mathrm{s}} \\
{[0 / 90 / 0 / 45 /-45]_{\mathrm{s}}} \\
{[0 / 90 / 0 / 45 /-45]_{\mathrm{s}}} \\
{[0 / 90 /-45 / 45]_{\mathrm{s}}}\end{array}$ \\
\hline
\end{tabular}




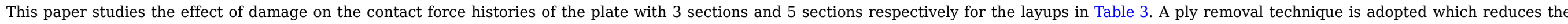

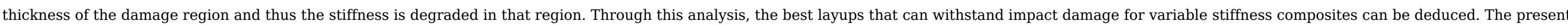

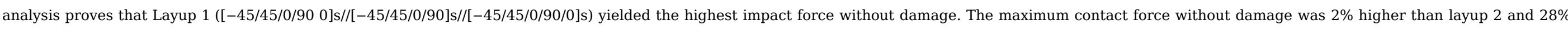

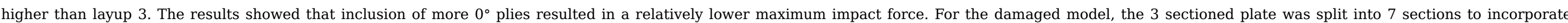

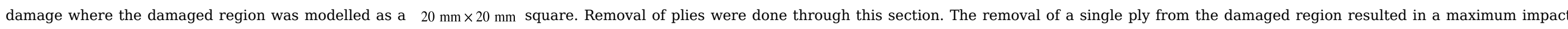

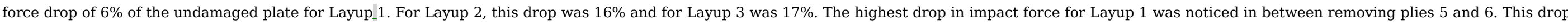

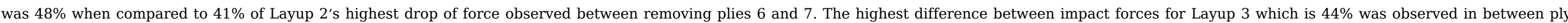

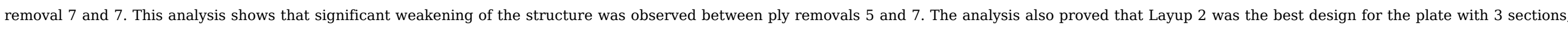
since the induced damage affected the impact force history lesser and mainly since it had a higher contact force after removing all plies from the damaged region (see Fig. 4).
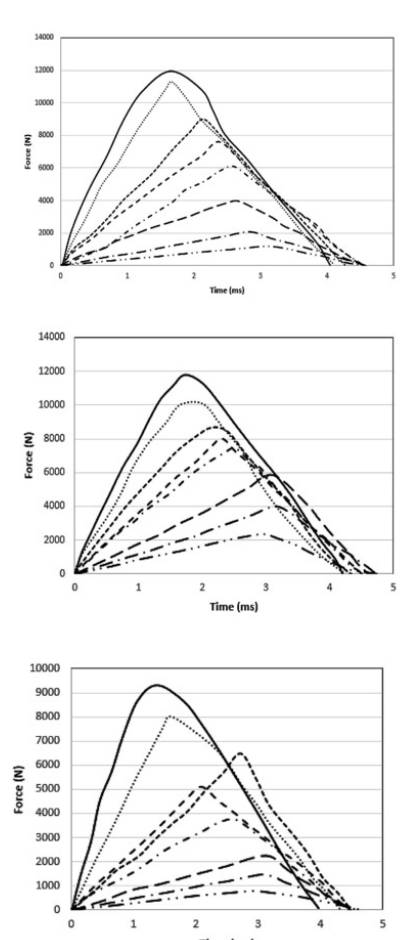

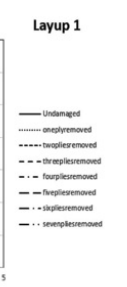

Layup 2

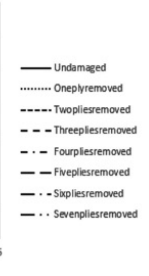

Layup 3

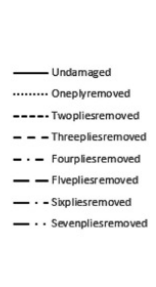

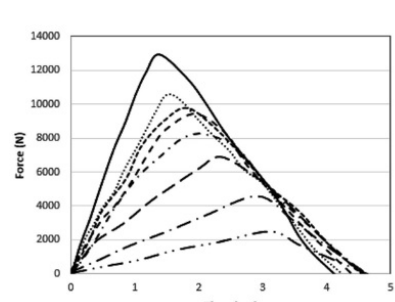

Layup 4

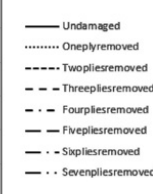

Layup 5

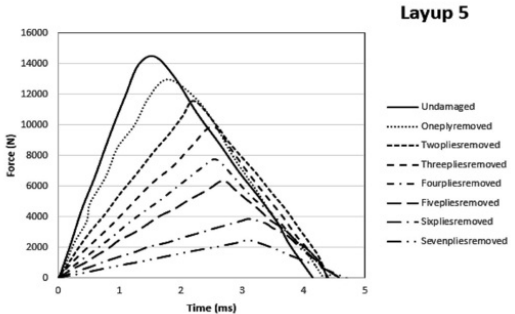

Layup 6

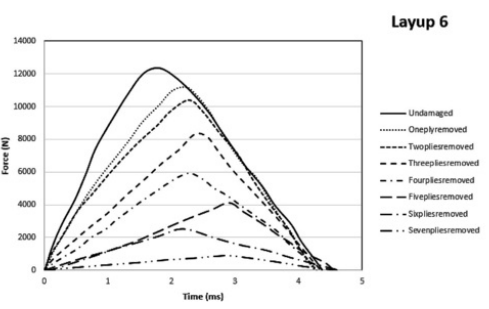

Fig. 4 Comparison of force - time history of undamaged and damaged models for various layups. 


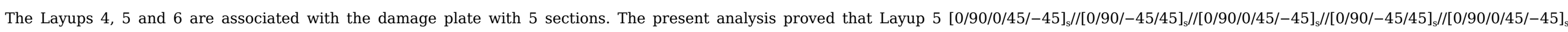

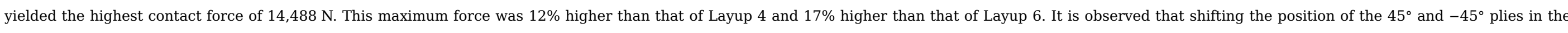

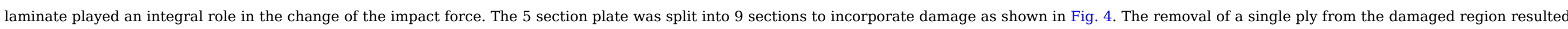

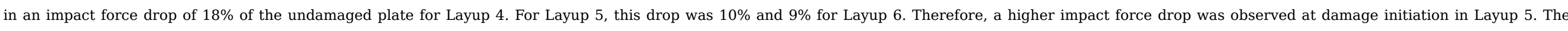

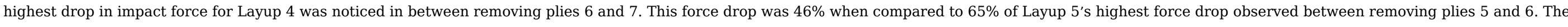

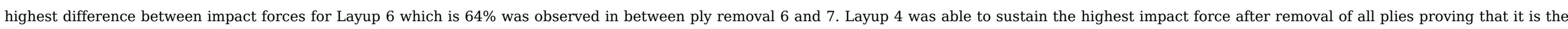
best layup design able to withstand damage even when Layup 5 yielded a higher impact force without damage induced (see Fig. 4).

\subsection{Explanation of damage behaviour}

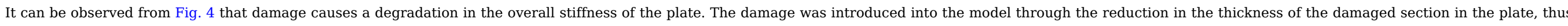

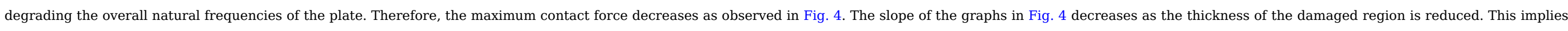

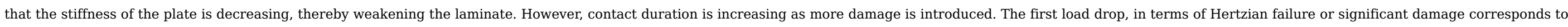

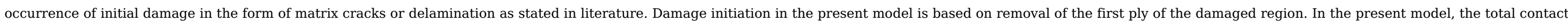
duration increases as the level of damage increases as shown in Table 4.

Table 4 Contact Time duration for the different layups.

\begin{tabular}{|c|c|c|c|c|c|c|c|}
\hline \multicolumn{8}{|c|}{ Contact time duration (ms) } \\
\hline Layups & 1ply removed & 2 plies removed & 3 plies removed & 4plies removed & 5 plies removed & 6 plies removed & 7plies removed \\
\hline 1 & 4.1 & 4.3 & 4.5 & 4.6 & 4.6 & 4.6 & 4.6 \\
\hline 2 & 4.2 & 4.3 & 4.5 & 4.5 & 4.6 & 4.7 & 4.7 \\
\hline 3 & 4.3 & 4.5 & 4.5 & 4.5 & 4.6 & 4.6 & 4.6 \\
\hline 4 & 4.2 & 4.4 & 4.5 & 4.6 & 4.6 & 4.7 & 4.7 \\
\hline 5 & 4.3 & 4.4 & 4.4 & 4.5 & 4.6 & 4.6 & 4.7 \\
\hline 6 & 4.3 & 4.3 & 4.4 & 4.4 & 4.6 & 4.6 & 4.7 \\
\hline
\end{tabular}

\subsection{Effect of curvature on damage behaviour}

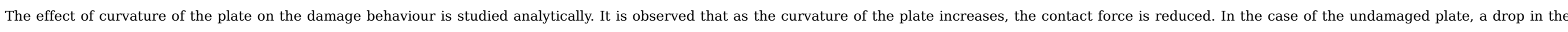

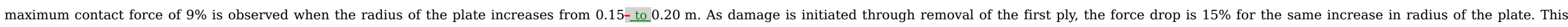
proves that the radius of the plate has an effect on the damage characteristics of the plate (see Fig. 5). 

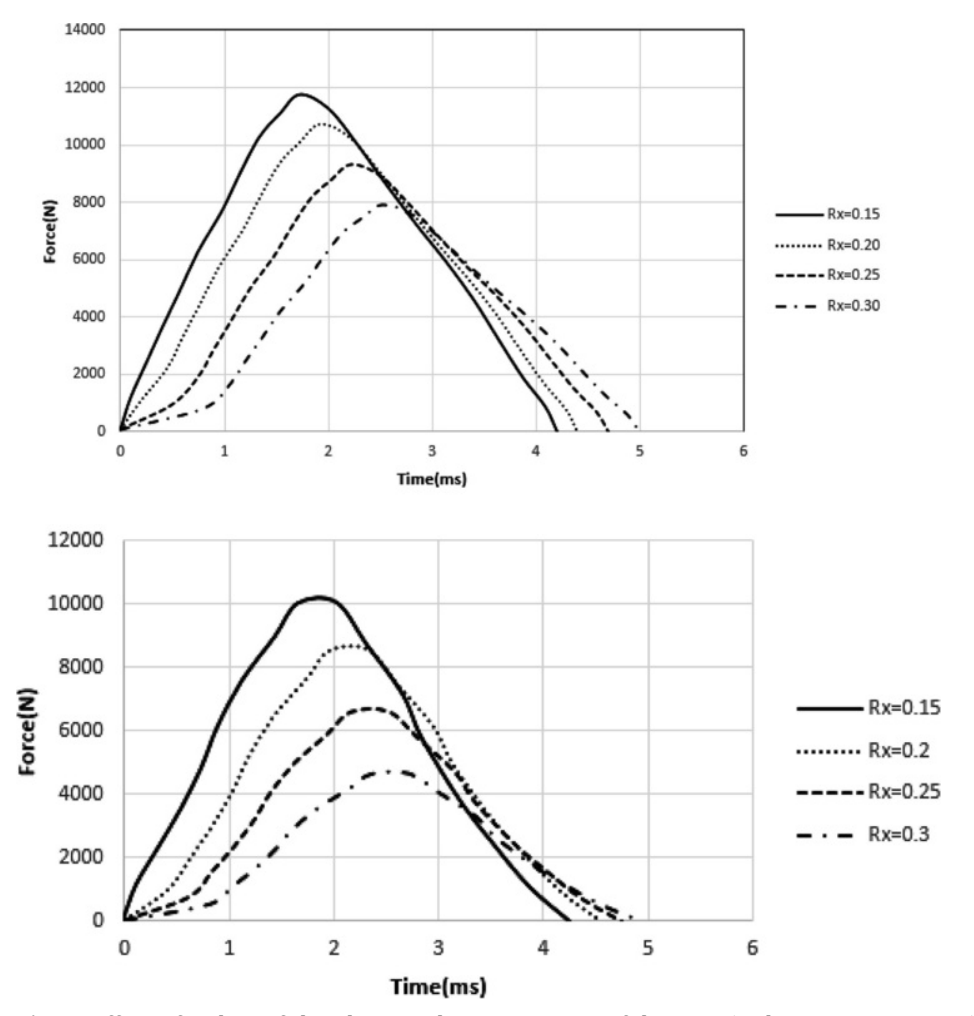

\section{Validation of theoretical model using FEM}

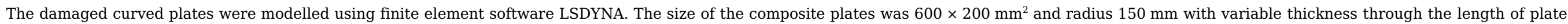

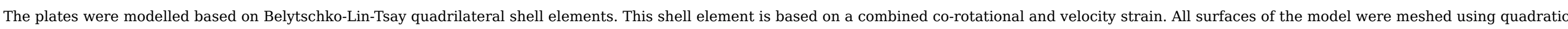

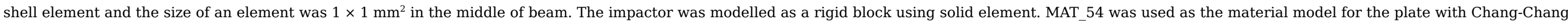

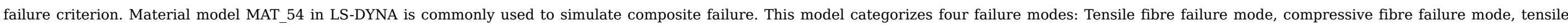

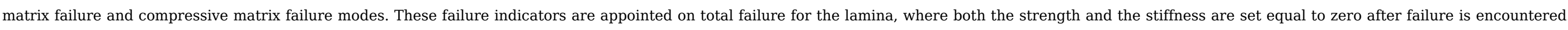

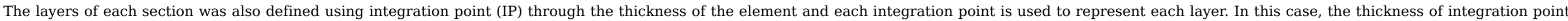

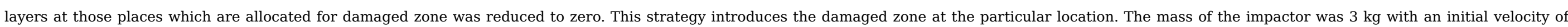
$6 \mathrm{~m} / \mathrm{s}$ (Fig. 6).

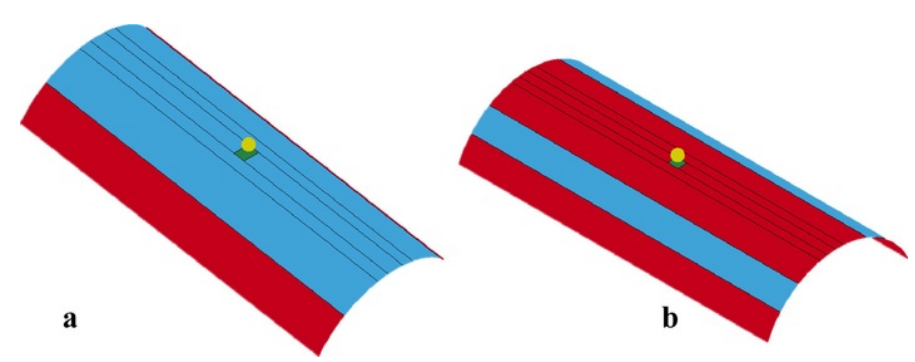




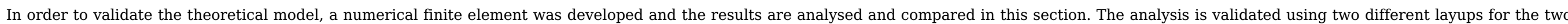

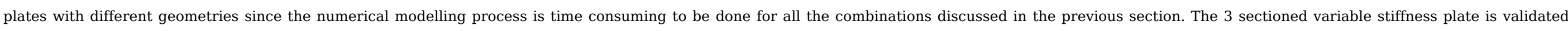

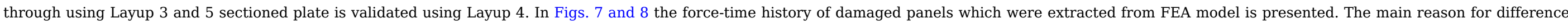

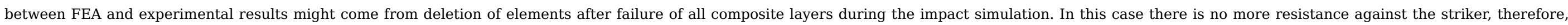
some discrepancies are observed between experimental and FEA results (see Fig. 9).
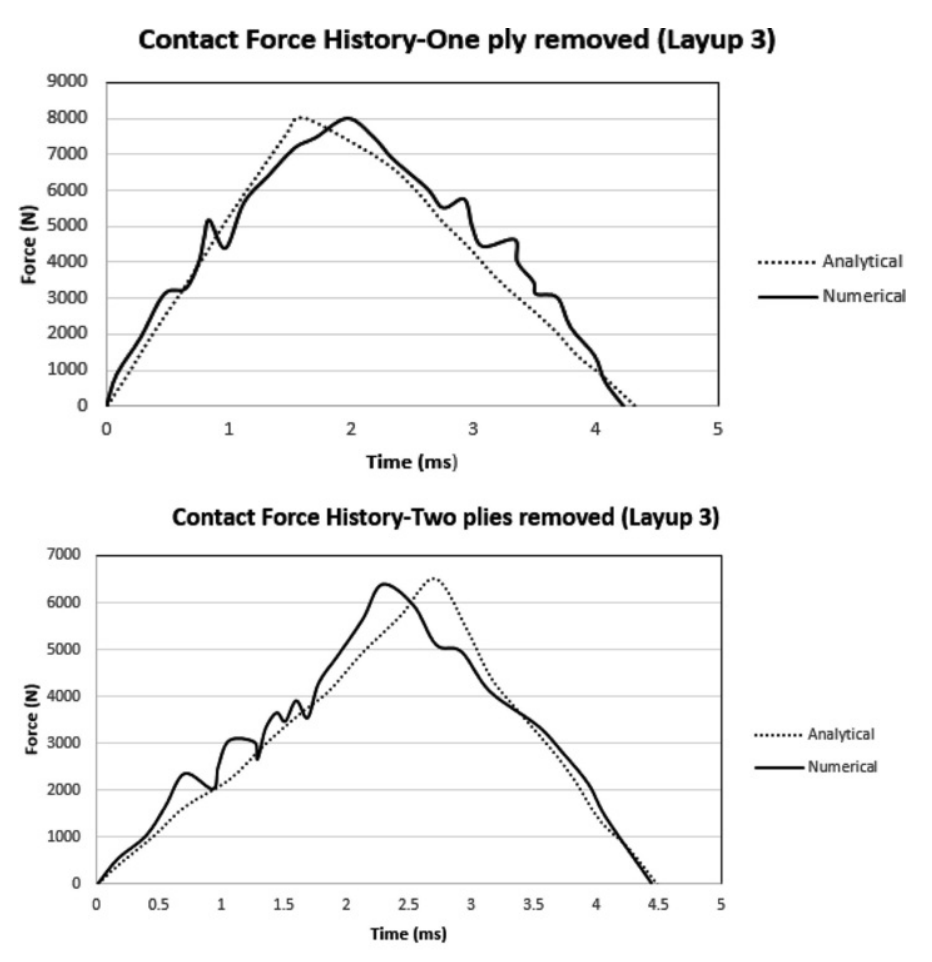

Contact Force History-Three plies removed (Layup 3)

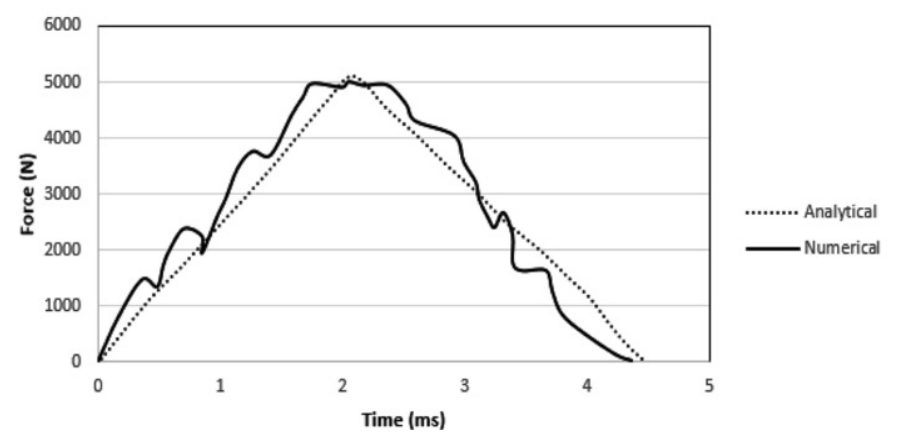

Fig. 7 Comparison between FE results and the developed theoretical model for layup 3. 
Contact Force History- One ply removed (Layup 4)

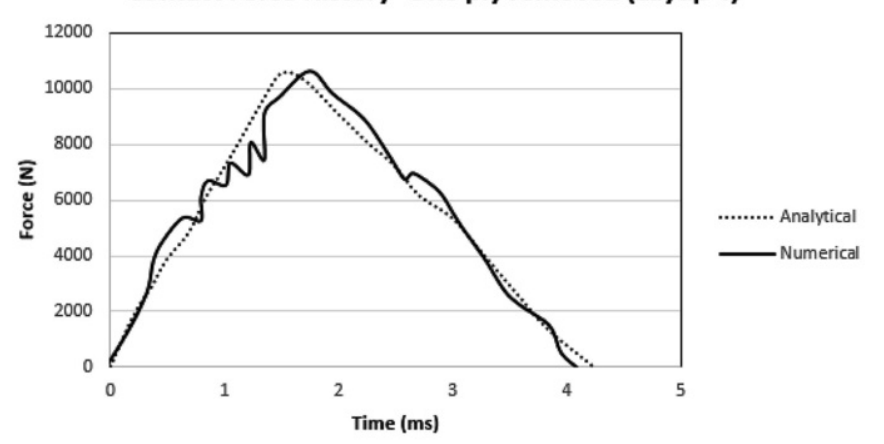

Contact Force History- Two plies removed (Layup 4)

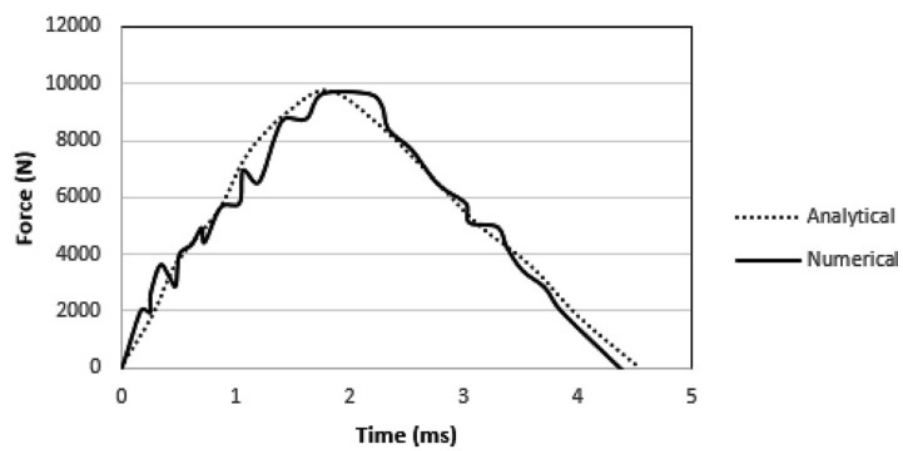

Contact Force History- Three plies removed (Layup 4)

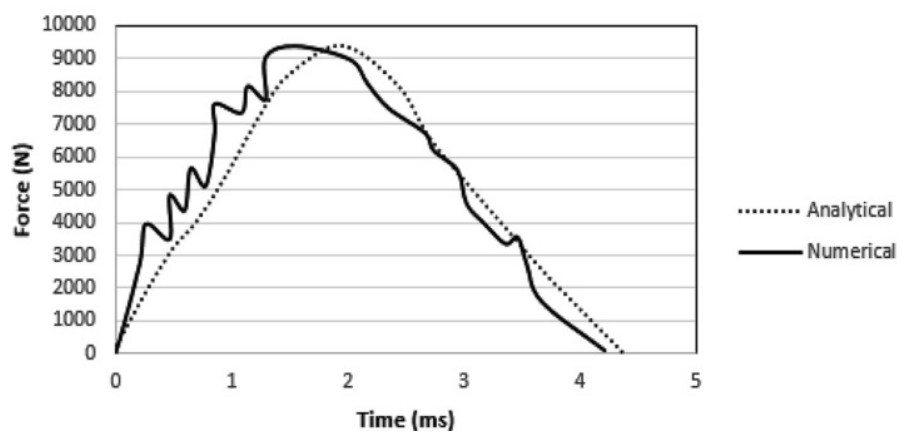

Fig. 8 Comparison between FE results and the developed theoretical model for layup 4. 


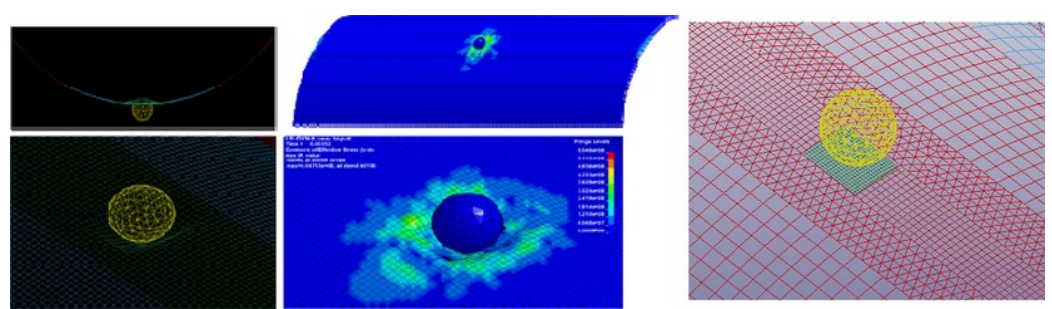

Fig. 9 Finite element images of damaged curved composite panels with variable stiffness a) element deformation, b) stress distribution and c) mesh generation.

\section{Conclusion}

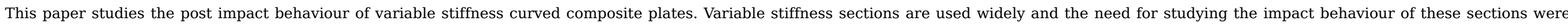

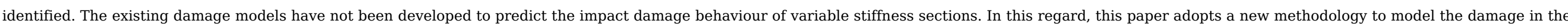

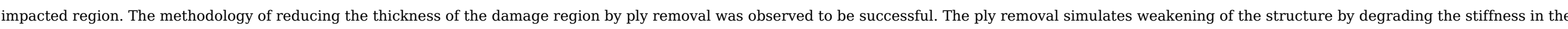

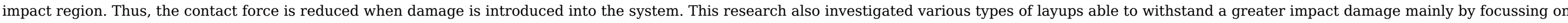

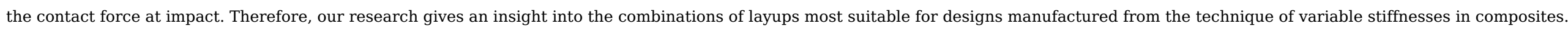

\section{References}

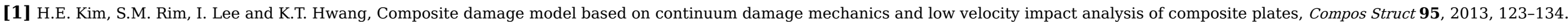

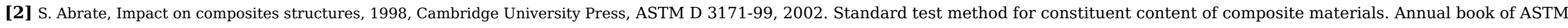
standards, West Conshohocken, PA..

[3] S. Gong, The elastic response of orthotropic laminates shells to low velocity impact, Compos Eng 4, 1994, 247-266.

[4] S.M.R. Khalili and A. Ardali, Low velocity impact response of doubly curved symmetric cross-ply laminated panel with embedded SMA wires, Compos Struct 2013, 216-226.

[5] L.S. Kistler and A.M. Waas, On the response of curved laminated panels subjected to transverse impact loads, Int J Solids Struct 36 (9), $1999,1311-1327$.

[6] H. Saghafi, G. Minak and A. Zucchelli, Effect of preload on the impact response of curved composite panels, Compos B Eng 60, $2014,74-81$.

[7] I.H. Choi, Geometrically nonlinear transient analysis of composite laminated plate and shells subjected to low-velocity impact, 2016, Compos Struct 142, 2016, 7-14.

[8] Z. Leylek, M.L. Scott, S. Georgiadis and R.S. Thomson, Computer modelling of impact on curved fibre composite panels, Compos Struct 47 (1-4), 1999 , 789-796.

[9] N.S. Goo and S.J. Kim, Dynamic contact analysis of laminated composite plates under low-velocity impact, AIAAJ 35 (9), $1997,1518-1521$.

[10] K.N. Shivakumar, W. Elber and W. Illg, Prediction of low-velocity impact damage in composite laminates, AIAAJ 23 (5), 1984, 442-449.

[11] H. Singh and P. Mahajan, Analytical modelling of low velocity large mass impact on composite plate including damage evolution, Compos Struct 149, $2016,79-92$.

[12] R. Olsson, Analytical prediction of large mass impact damage in composite laminates, Compos Part A Appl Sci Manuf 32 (9), $2001,1207-1215$.

[13] R. Olsson, Analytical model for delamination growth during small mass impact on plates, Int J Solids Struct 47 (21), $2010,2884-2892$.

[14] R. Olsson, Analytical prediction of damage due to large mass impact on thin ply composites, Compos Part A Appl Sci Manuf 72, 2015, 184-191.

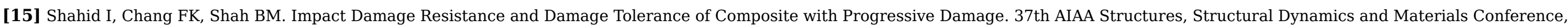
1996: pp. 766-775. 


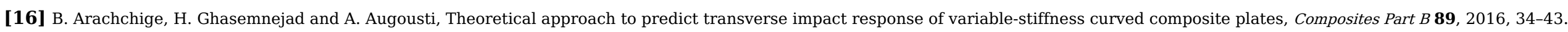

\section{Queries and Answers}

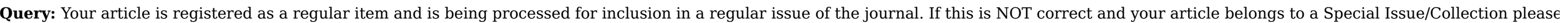
contact s.priya.1@elsevier.com immediately prior to returning your corrections.

Answer: Correct.

Query: The author names have been tagged as given names and surnames (surnames are highlighted in teal color). Please confirm if they have been identified correctly. Answer: Confirm.

Query: Please note that Figs. 2 and 6 are not cited in the text. Please check that the citations suggested by the copyeditor are in the appropriate place, and correct if necessary. Answer: The suggestions are correct. 
2017-01-18

Post impact analysis of damaged

\section{variable-stiffness curved composite plates}

Arachchige, Buddhi

Elsevier

B. Arachchige, H. Ghasemnejad, Post impact analysis of damaged variable-stiffness curved composite plates, Composite Structures, Vol. 166, 15 April 2017, pp. 12-21

https://dspace.lib.cranfield.ac.uk/handle/1826/11436

Downloaded from Cranfield Library Services E-Repository 